\title{
Análise bibliométrica e sistêmica da literatura sobre viabilidade econômica-financeira, gestão e sustentabilidade da reciclagem de resíduos da construção civil
}

\section{A bibliometric and sistemic analysis on economic feasibility, management and sustainability of waste construction}

Luis Carlos Pais Gularte ${ }^{1}$

Sandro César Bortoluzzi²

José Donizetti de Lima ${ }^{3}$

Michele Aparecida Nepomuceno Pinto ${ }^{4}$

Andréia dos Santos Goffi ${ }^{5}$

1 Mestre em Engenharia de Produção e Sistemas pela Universidade Tecnológica Federal do Paraná (UTFPR).

Economista, Técnico Administrativo em Educação

na Universidade Federal da Fronteira Sul (UFFS). Universidade Tecnológica Federal do Paraná luisgularte755@hotmail.com

2 Docente do Programa de Pós-Graduação em Engenharia de Producão e Sistemas pela Universidade Tecnológica Federal do Paraná (UTFPR). Doutor em Engenharia de Produção pela Universidade Federal de Santa Catarina (UFSC). Universidade Tecnológica Federal do Paraná scbortoluzzi@gmail.com

3 Docente do Programa de Pós-Graduação em Engenharia de Produção e Sistemas pela Universidade Tecnológica Federal do Paraná (UTFPR). Doutor em Engenharia de Produção pela Universidade Federal do Rio Grande do Sul (UFRGS). Universidade Tecnológica Federal do Paraná donizetti@utfpr.edu.br

4 Doutoranda em Economia na Universidade Estadual de Maringá (UEM). Economista, Técnica Administrativa em Educação na Universidade Federal da Fronteira Sul (UFFS). Universidade Estadual de Maringá mi_nepomuceno@hotmail.com

5 Mestre em Engenharia de Produção e Sistemas pela Universidade Tecnológica Federal do Paraná (UTFPR). Universidade Tecnológica Federal do Paraná andreiagoffi@gmail.com

\section{Resumo}

A literatura a respeito da viabilidade econômico-financeira, gestão e sustentabilidade na reciclagem de resíduos da Construção Civil é bem abrangente. Dessa forma, este estudo tem como objetivo formar um Portfólio Bibliográfico com os principais artigos que tratam do tema, buscando identificar lacunas que sejam passíveis de se tornarem futuras pesquisas. Para isso, realizou-se um processo de seleção e análise da literatura, por meio do procedimento ProKnow-C, o qual identificou 21 artigos para compor o Portfólio Bibliográfico, os quais passaram por análises bibliométrica, com dados estatísticos, e sistêmica a partir de quatro lentes que estão ligadas a afiliação teórica dos autores.

Palavras-chaves: ProKnow-C. Viabilidade econômico-financeira. Construção Civil. Análise bibliométrica. Análise sistêmica.

\section{Abstract}

The literature on the economic and financial viability, management and sustainability in waste recycling Construction is very comprehensive. Thus, this study aims to form a Bibliographical portfolio with key articles dealing with the issue in order to identify gaps that are likely to become future research. For thiswas held a process of selection and analysis of the literature, through ProKnow-C procedure, which identified 21 items to compose the Bibliographical Portfolio, which passed through bibliometric analysis with statistical data, and systemic from the four lenses which are connected to theoretical affiliation of the author.

Keywords: ProKnow-C. Economic and financial viability. Construction. Bibliometric analysis. Systemic analysis. 


\section{Introdução}

Uma preocupação crescente quando se fala em decisão de investimento nas empresas do mundo contemporâneo é considerar a maior gama possível de fatores que podem influenciar na viabilidade ou não de se investir em determinado projeto. Atualmente, há inúmeras maneiras de quantificar essa viabilidade, que pode ser analisada por meio do foco econômico-financeiro, ambiental, social, dentre outros, variando de acordo com o interesse da empresa (Pinto, 1999).

Entretanto, usualmente, as empresas buscam maximizar seus lucros, de forma que a análise da viabilidade econômico-financeira acaba sendo o norteador na hora da decisão do investimento. Essa é umas das razoes pelas quais os estudos de viabilidade econômico-financeira devem ser tratados como prioridade pelos tomadores de decisão e devem buscar seremos mais próximos possíveis da realidade, para evitar surpresas futuras (Nunes, 2004).

Um projeto de investimento que busca implantar uma Usina de Reciclagem de Resíduos da Construção Civil e Demolição (URRCD) também requer que se faça um estudo prévio da viabilidade econômico-financeira do investimento, além de análises da gestão e sustentabilidade da mesma. Desse modo, obter-se-ia um panorama global do investimento de forma que se torna possível tomar a decisão de implantá-la (Harzer, 2015).

A reciclagem de Resíduos da Construção Civil e Demolição (RCD) é uma prática que apresenta vantagens ambientais à sociedade, tais como: economia na aquisição de matérias-primas, decréscimo da poluição gerada pelo entulho e de suas consequências, como enchentes e assoreamento de rios e córregos, preservação e redução das áreas de aterros inertes (Marques, 2005).

Porém, conforme destacam Santos e Pompeu (2014), para o meio empresarial, o argumento referindo-se somente a questões ambientais ou sustentáveis se torna insuficiente, principalmente na atividade proposta, pois a instalação de uma URRCD demanda investimentos iniciais elevados. Portanto, torna-se necessário, acima de tudo, um planejamento econômico-financeiro para testar a viabilidade de um empreendimento deste porte. É necessário justificar a reciclagem desses resíduos, como uma atividade que, entre outros aspectos, gere emprego e renda e que seja lucrativa economicamente.

Azevedo, Kiperstok e Moraes (2006) afirmam que a geração de RCD pode ocorrer nas diferentes fases do ciclo de vida dos empreendimentos: construção, manutenção, reformas e demolição. De acordo com dados da ABRELPE (2014), a geração estimada de RCD no Brasil seria em torno de 122.262 toneladas por dia. Esses dados mostram o elevado potencial que o setor tem em produzir agregados reciclados, os quais podem agregar valor à produção. Com isso, há diminuição da necessidade de matérias-primas virgens, provocando redução nos custos das empresas e também causando impacto socioambiental positivo, uma vez que esses resíduos deixam de serem descartados em aterros ou até no próprio meio ambiente (Pinto, 1999).

Considerando esse cenário, é visível a necessidade de se estudar esse tema mais profundamente. Entretanto, há uma infinidade de trabalhos publicados anualmente com a temática da reciclagem de RCD, sendo que nem todos abordam a questão da gestão, sustentabilidade ou viabilidade econômico-financeira desses empreendimentos, de forma que se faz necessário selecionar um portfólio de publicações que garanta artigos cientificamente válidos, com conteúdos alinhados ao tema de pesquisa e que possam servir de base para futuras pesquisas a respeito do tema.

Frente ao contexto exposto, surge o questionamento de pesquisa, o qual norteia a elaboração desse trabalho científico: como construir 
o conhecimento inicial em um pesquisador sobre o tema gestão, sustentabilidade e viabilidade econômico-financeira da implantação de uma URRCD privada para a destinação correta dos RCD, por meio da utilização de uma metodologia de pesquisa estruturada?

Desta forma, o presente trabalho tem como objetivo, por meio da construção do conhecimento no pesquisador, identificar lacunas passíveis de se tornarem futuras pesquisas a respeito da gestão, sustentabilidade e viabilidade econômico-financeira da implantação de uma URRCD privada para a destinação correta dos RCD. Os objetivos específicos deste trabalho são, a partir do método Proknow-C, montar um portfólio de artigos que abranja os três eixos definidos na pesquisa e, a partir deste portfólio, realizar uma análise bibliométrica e sistêmica do mesmo.

A motivação do interesse no tema da pesquisa está alicerçada na carência de procedimentos que envolvam as áreas de gestão, sustentabilidade e viabilidade econômica de resíduos da Construção Civil. Além de corroborar com pesquisadores que pretendem desenvolver pesquisas com o tema voltado a esta área do conhecimento, formando um arcabouço de produções a fim de auxiliar o início das pesquisas. Este estudo, após esta seção introdutória, apresenta, em sua segunda seção, a metodologia aplicada, na terceira seção são feitas as análises bibliométrica e sistêmica e, por último, encontram-se as considerações finais e referências.

\section{Procedimentos metodológicos}

\subsection{Procedimentos para seleção de artigos do Portfólio Bibliográfico}

Essa seção apresentará os procedimentos utilizados para a seleção dos artigos que comporão o Portfólio de artigos da presente pesquisa. A partir deste Portfólio Bibliográfico, faz-se a análise bibliométrica, que consiste em identificar os periódicos com maior participação, os autores de maior relevância e as palavras-chaves mais utilizadas, e a análise sistêmica, que busca evidenciar para cada lente, a perspectiva estabelecida, os destaques e as oportunidades de conhecimentos encontrados na amostra (ENSSLIN, ENSSLIN, \& SOUZA., 2014).O instrumento de intervenção utilizado é o processo para construir conhecimento a partir dos interesses e delimitações de pesquisadores, segundo a visão construtivista, Knowledge Development Process-Constructivist (ProKnow-C), presente em trabalhos como os de Ensslin et al.(2014); Bortoluzzi, Ensslin e Ensslin (2011); Bortoluzzi, Ensslin, Ensslin e Chaves (2014); Lacerda, Ensslin \& Ensslin (2012); Semler, Bortoluzzi \& Schenatto (2015); Alves e Ensslin (2012).

Para iniciar a pesquisa algumas restrições foram estabelecidas: a primeira foi a respeito do tipo de documento utilizado na pesquisa: foram considerados somente artigos, pois estes passaram por um crivo técnico. A segunda foi a data de publicação: foram considerados os documentos publicados entre 2000 e 2015. A terceira restrição consiste nas bases de dados consultadas: foram incluídas as bases de dados Scopus e ISI Web of Knowledge, sendo ambas indexadas pela CAPES e com enquadramento teórico com o eixo de pesquisa. Por fim, a quarta restrição foi a de palavras-chaves: para definir as palavras-chaves foram inicialmente estabelecidos os eixos de pesquisa e a partir destes estabeleceu-se as combinações que os contemplassem. Os eixos foram definidos como: (i) viabilidade econômico-financeira; (ii) gestão de resíduos da Construção Civil; e (iii) sustentabilidade. $\mathrm{O}$ primeiro representa o tema central da pesquisa e os outros dois tratam do desempenho das usinas e seus impactos à sociedade e ao meio ambiente. 
Associou-se as palavras-chaves do eixo de Viabilidade Econômico-Financeira (economic feasibility, economic viability, Monte Carlo simulation), com as palavras relacionadas ao eixo de pesquisa Gestão de Resíduos da Construção Civil (waste, construction, recycling, demolition, management) e com as palavras do terceiro eixo: Sustentabilidade (reverse logistics, plant, facility, C\&́D, Sustainability, aggregate) formando um conjunto de 90 combinações.

De posse das palavras-chaves e da base que se caracteriza pelo campo amostral, iniciou-se o processo de seleção dos artigos que comporão o portfólio para a construção do referencial teórico da pesquisa em pauta. Utilizando as palavras-chaves anteriormente definidas, a busca na base de dados ISI retornou uma massa de 4.415 referências e a base de dados SCOPUS encontrou 5.892 referências, totalizando 10.307 arquivos.

A partir desse primeiro banco de arquivos, partiu-se para a etapa de filtragem do banco de artigos, a qual, Conforme Knoff, Lacerda, Ensslin \& Ensslin (2014), é subdividida em cinco fases, sendo elas: (i) eliminação de arquivos que não eram artigos e de artigos repetidos e/ou com data de publicação inferior ao ano 2000; (ii) alinhamento pela leitura do título; (iii) alinhamento quanto ao reconhecimento científico; (iv) alinhamento pela leitura do resumo; e (v) alinhamento pela leitura integral dos artigos.Para um efetivo gerenciamento dessas referências, elas foram importadas para o software Endnote ${ }^{\circledR}$. Com o auxílio dessa ferramenta, foi aplicado o primeiro filtro, excluindo da amostra as referências que não condiziam com os objetivos da pesquisa: 2.281 arquivos que não eram artigos (papers), 3.012 referências duplicadas e 1.675 artigos com datas anteriores ao ano 2000. Uma vez realizadas essas 7.868 exclusões, a biblioteca de artigos passou a ter 2.439 artigos.

Com essas 2.439 referências, passou-se à leitura dos títulos dos artigos para observar o ali- nhamento desses com a presente pesquisa. Depois dessa análise, 2.004 referências foram excluídas por não terem alinhamento com a pesquisa, restando, dessa forma, 435 referências a serem analisadas. As 435 referências que, pelos seus títulos, se alinhavam com o tema de pesquisa, foram analisadas pelo seu reconhecimento científico desde sua publicação. Para realizar essa análise, todas as referências foram consultadas pela ferramenta Google Scholar (2015) quanto ao número de citações e ordenadas de forma decrescente.

De posse dessas informações, foi estabelecido um valor de corte para os artigos mais citados. O ponto de corte estabelecido foi de quatro citações, pois este grupo representa $97 \%$ de todas as citações obtidas pelos 435 artigos em análise. Segundo Lacerda et al. (2012), se a pesquisa selecionar a minoria dos artigos mais citados, esses representam a maioria do reconhecimento científico presente no conjunto atual de artigos.

Com esse terceiro filtro aplicado, o banco de dados passou a ter 203 artigos. Os outros 232 artigos que estavam abaixo dessa linha de corte foram classificados, nessa pesquisa, como "artigos com reconhecimento científico ainda não confirmado". Vale ressaltar que esses artigos menos citados ainda passam por um processo de análise sob outros critérios, pelos quais podem ainda fazer parte do portfólio final de artigos que constituirá parte do referencial teórico da pesquisa (Lacerda et al., 2012).

O próximo filtro aplicado é o do alinhamento pela leitura do resumo. Foram analisados os resumos (abstract) dos 203 artigos selecionados, para verificar seu alinhamento ao foco da pesquisa em questão. A partir da leitura dos resumos, 157 artigos foram excluídos devido à falta de alinhamento, restando 46 artigos que foram adicionados ao Repositório A. Os autores desses artigos também foram identificados para compor o banco de autores para uso posterior. 
Seguindo o processo estabelecido no ProKnow-C, os 232 artigos com reconhecimento científico ainda não confirmados foram divididos entre os artigos publicados há dois anos ou menos (80 artigos) e os artigos com mais de dois anos de publicação (152 artigos). Dentro desse grupo de 152 artigos com mais de dois anos, buscou-se aqueles cujos autores eram os mesmos que estavam presentes no banco de autores dos artigos mais representativos. Identificou-se um total de 24 artigos, os quais foram juntados aos 80 recentes, totalizando 104 artigos que passaram pelo filtro do alinhamento de seus resumos. Nesse filtro, 82 artigos foram eliminados e 22 artigos foram selecionados nesse processo de reanálise, os quais passaram a compor o Repositório B.

Dessa forma, somando os artigos do Repositório A com os do Repositório B, obtevese um total de 68 artigos, os quais passaram pelo último filtro: quanto ao alinhamento pela leitura do texto integral. O primeiro passo foi verificar se o artigo estava disponível na íntegra no portal da CAPES. Nesse momento 8 artigos foram excluídos, restando 60 para leitura completa. Após a leitura, os artigos foram separados entre os alinhados ao tema da pesquisa e os desalinhados, de forma que 39 artigos foram excluídos por estarem desalinhados e os 21 artigos alinhados passaram a compor o Portfólio Bibliográfico, nomeados, no Quadro 01, por ordem alfabética do primeiro autor.

\subsection{Procedimentos para análise bibliométrica e de conteúdo (sistêmica)}

Segundo Ensslin, Ensslin, Lacerda \& Tasca (2010), a Análise Bibliométrica é um processo de evidenciação quantitativa dos dados estatísticos de um conjunto definido de artigos (Portfólio Bibliográfico) para a gestão da informação e do conhecimento científico de um dado assunto, realizado por meio da contagem de documentos.
Por meio dessa análise traça-se o perfil das publicações do portfólio e de suas referências no que se refere a quais periódicos que mais publicaram sobre o tema, quais os autores de maior destaque, quais os artigos com maior reconhecimento científico e as palavras-chaves mais utilizadas (Knoff et al., 2014). A primeira parte desse processo é realizar a análise bibliométrica dos 21 artigos selecionados, em seguida são analisadas as referências dos artigos selecionados e, por fim, são apresentados os resultados da classificação dos artigos conforme relevância acadêmica na amostra.

Já a análise sistêmica é um processo científico utilizado para, a partir de uma visão de mundo (filiação teórica) explicitada por suas lentes, analisar uma amostra de artigos representativa de um dado assunto de pesquisa, visando evidenciar os destaques e as oportunidades de conhecimentos encontrados na amostra (Ensslin et al., 2010; Lacerda et al., 2012).

A Análise Sistêmica utiliza-se de abordagens denominadas lentes, apresentadas no Quadro 02. Além de identificar os destaques (pontos fortes) e as lacunas (oportunidades) de conhecimento presente nos artigos analisados, buscou-se verificar se os mesmos estão alinhados com a afiliação teórica adotada pelos autores (Ensslin et al., 2010; Nagaoka, Ensslin, Ensslin \& Nagaoka, 2011). Destaca-se que a análise sistêmica do ProKnow-C original é com outras lentes, dessa forma, o método foi adaptado para que se atendam os objetivos da presente pesquisa.

\section{Apresentação e discussão dos resultados}

\subsection{Análise Bibliométrica}

Conforme destacado na metodologia, a análise bibliométrica fará uma análise quantitativa dos dados estatísticos dos artigos selecionados 
AJAYI, S. O., OYEDELE, L. O., BILAL, M., AKINADE, O. O., ALAKA, H. A., OWOLABI, H. A., KADIRI, K. O. (2015). Waste effectiveness of the construction industry: Understanding the impediments and requisites for improvements. Resources, Conservation and Recycling, v. 102, p. 101-112.

BANIAS, G., ACHILlAS, C., VlACHOKOSTAS, C., MOUSIOPOULOS, N., TARSENIS, S. (2010). Assessing multiple criteria for the optimal location of a construction and demolition waste management facility. Building and Environment, v. 45, n. 10, p. $2317-26$.

BEGUM, R. A., SIWAR, C., PEREIRA, J. J., JAAFAR, A. H. (2006). A benefit-cost analysis on the economic feasibility of construction waste minimization: the case of Malaysia. Resources, Conservation and Recycling, v. 48, n. 1, p. 86-98.

BEGUM, R. A., SIWAR, C., PEREIRA, J. J., JAAFAR, A. H. (2009). Attitude and behavioral factors in waste management in the construction industry of Malaysia. Resources, Conservation and Recycling, v. 53, n. 6, p. $321-328$.

COELHO, A., BRITO, J. (2012). Influence of construction and demolition waste management on the environmental impact of buildings. Waste Management, v.32, n.3, p.532-41.

COELHO, A., BRITO, J. (2013a). Economic viability analysis of a construction and demolition waste recycling plant in Portugal - part I: location, materials, technology and economic analysis. Journal of Cleaner Production, v. 39, p. 338-352.

COELHO, A., BRITO, J. (2013b). Economic viability analysis of a construction and demolition waste recycling plant in Portugal - part II: economic sensitivity analysis. Journal of Cleaner Production, v. 39, p. 329-337.

DURAN, X., LENIHAN, H., O'REGAN, B. (2006). A model for assessing the economic viability of construction and demolition waste recycling - the case of Ireland. Resources, Conservation and Recycling, v. 46, n. 3, p. 302-320.

FATTA, D., PAPADOPOUlOS, A., AVRAMIKOS, E., SGOUROU, E., MOUSTAKAS, K., KOURMOUSSIS, F., LOIZIDOU, M. (2003). Generation and management of construction and demolition waste in Greece - an existing challenge. Resources, Conservation and Recycling, v. 40, n. 1, p. 81-91.

HOSSEINI, M. R., RAMEEZDEEN, R., CHILESHE, N., LEHMANN, S. (2015). Reverse logistics in the construction industry. Waste Management \& Research, v. 33, n.6, p. 499-514.

KARTAM, N., AL-MUTAIRI, N., AL-GHUSAIN, I., AL-HUMOUD, J. (2004). Environmental management of construction and demolition waste in Kuwait. Waste Management, v. 24, n. 10, p. 1049-1059.

KASTHURBA, A., REDDY, K., REDDY, D. V. (2014). Sustainable Approaches for Utilizing Waste in Building Construction: Two Case Studies in India. International Journal of Earth Sciences and Engineering, v. 7, n. 3, p. 838-844.

LIU, J., WANG, Y. (2013). Cost Analysis of Construction and Demolition Waste Management: Case Study of the Pearl River Delta of China. Open Construction and Building Technology Journal, v. 7, p. 251-263.

NAGALLI, A. (2013). The Sustainability of Brazilian Construction and Demolition Waste Management System. The Electronic Journal of Geotechnical Engineering, v. 18, p. 1755-59.

NUNES, K. R. A., MAHLER, C. F., VALLE, R., NEVES, C. (2007). Evaluation of investments in recycling centers for construction and demolition wastes in Brazilian municipalities. Waste Management, v. 27, n. 11, p. 1531-1540.

RODRÍGUEZ, G., ALEGRE, F. J., MARTíNEZ, G. (2007). The contribution of environmental management systems to the management of construction and demolition waste: The case of the Autonomous Community of Madrid (Spain). Resources, Conservation and Recycling, v. 50, n. 3, p. 334-349.

SROUR, I. M., CHEHAB, G. R., EL-FADEL, M., TAMRAZ, S. (2013). Pilot-based assessment of economics of recycling construction demolition waste. Waste Management \& Research, v. 31, n. 11, p. 1170-79.

TAM, V. W., KOTRAYOTHAR, D., LOO, Y. C. (2009). On the prevailing construction waste recycling practices: a South East Queensland study. WasteManagement $\mho$ Research, v. 27, n. 2, p. 167-174.

WANG, J. Y., TOURAN, A., CHRISTOFOROU, C., FADLALLA, H. (2004). A systems analysis tool for construction and demolition wastes management. Wastemanagement, v. 24, n. 10, p. 989-997.

YEHEYIS, M., HEWAGE, K., ALAM, M.S., ESKICIOGLU, C., SADIQ, R. (2013). An overview of construction and demolition waste management in Canada: a lifecycle analysis approach to sustainability. Clean Technologies and Environmental Policy, v.15, n.1, p. 81-91.

ZHAO, W., LEEFTINK, R. B., ROTTER, V. S. (2010). Evaluation of the economic feasibility for the recycling of construction and demolition waste in China - The case of Chongqing. Resources, Conservation and Recycling, v. 54, n. 6, p. 377-389.

Quadro 01: Artigos que formam o Portfólio Bibliográfico

Fonte: Elaborado pelos autores. 


\begin{tabular}{|c|c|}
\hline Lente & Finalidade \\
\hline $\begin{array}{c}\text { Abordagem da } \\
\text { pesquisa }\end{array}$ & $\begin{array}{c}\text { Qual o tipo de análise feita para } \\
\text { atingir o objetivo da pesquisa? } \\
\text { Qual abordagem foi utilizada } \\
\text { pelo pesquisador? }\end{array}$ \\
\hline $\begin{array}{c}\text { 2 - Método de } \\
\text { pesquisa }\end{array}$ & $\begin{array}{c}\text { Qual método de pesquisa foi } \\
\text { utilizado? }\end{array}$ \\
\hline 3 - Metodologia & $\begin{array}{c}\text { Quantos trabalhos utilizam os } \\
\text { indicadores de viabilidade } \\
\text { econômica, tais como TIR, VPL } \\
\text { e Payback? Houve utilização } \\
\text { da análise de sensibilidade } \\
\text { e da análise de cenários? Foi } \\
\text { considerada a incerteza no } \\
\text { processo de decisão por meio } \\
\text { da Simulação de Monte Carlo? } \\
\text { Foi empregada a flexibilidade } \\
\text { gerencial? }\end{array}$ \\
\hline $\begin{array}{c}4 \text { - Gestão e } \\
\text { Sustentabilidade }\end{array}$ & $\begin{array}{c}\text { O artigo propõe novas formas } \\
\text { de analisar o processo de } \\
\text { gestão de resíduos? }\end{array}$ \\
\hline
\end{tabular}

Quadro 2: Lentes da análise sistêmica da metodologia ProKnow-C

Fonte: Elaborado pelos autores.

para o Portfólio Bibliográfico. Essa análise foi dividida em duas partes: primeiro é feita a análise bibliométrica dos 21 artigos selecionados e, em um segundo momento, são analisadas as referências desses artigos selecionados e apresentados os resultados da classificação dos artigos conforme relevância acadêmica na amostra.

\subsubsection{Análise Bibliométrica dos Artigos Selecionados}

Nessa etapa são considerados os 21 artigos do Portfólio bibliográfico e a análise destes é feita por intermédio do grau de relevância dos periódicos em que foram publicados, do reconhecimento científico dos artigos, do grau de relevância dos autores e das palavras-chaves mais utilizadas.

A relevância dos periódicos mostrou que o periódico Resources, Conservation and Recycling se destaca ao ter publicado 7 artigos do Portfólio Bibliográfico, seguido pelo periódico Waste Management, com 4 artigos, e Waste Management and Research, com 3 artigos, em seguida vem o Journal of Cleaner Production com duas publicações. Os demais periódicos contaram com apenas um artigo cada.

Considerando o número de citações por artigo, há poucos artigos com a maioria das citações. No presente estudo, verificou-se que apenas treze artigos detêm mais de $80 \%$ das citações do Portfólio Bibliográfico, sendo que os destaques são: Kartam et al. (2004) com 14\% do total de citações; Begum et al. (2006) e Fatta et al. (2003) com $12 \%$ do total de citações cada.

Quanto à quantidade de artigos escritos por autor, verificou-se que há dois autores que se destacam em relação aos demais por terem contribuído com três artigos cada (Brito, J. e Coelho, A.) e cinco autores que têm dois trabalhos cada entre os selecionados para compor o Portfólio Bibliográfico (Begum, R. A.; Jaafar, A. H.; Pereira, J. J.; Siwar, C.; Wang, J. Y.). Os demais participaram com apenas um artigo.

No que diz respeito às palavras-chaves mais utilizadas no Portfólio Bibliográfico, a Figura 01 mostra que, das 14 palavras-chaves utilizadas nesta pesquisa, nove também estavam entre as palavras-chaves mais citadas pelos artigos do Portfólio Bibliográfico.

\subsubsection{Análises bibliométrica das referências dos artigos selecionados}

Nesta seção são analisados os 246 artigos citados como referências bibliográficas dos 21 artigos do Portfólio Bibliográfico. A Figura 2 apresenta a relevância dos periódicos nas referências dos artigos no Portfólio Bibliográfico. Verifica-se que os dois periódicos com mais artigos nas referências são os que também tiveram mais artigos no Portfólio Bibliográfico desta pesquisa, os quais são Resources, Conservation and Recycling e Waste Management. Houve, também, a ocorrência de dezessete periódicos que apresentaram apenas uma referência cada, de forma que não foram expostos na Figura 2. 


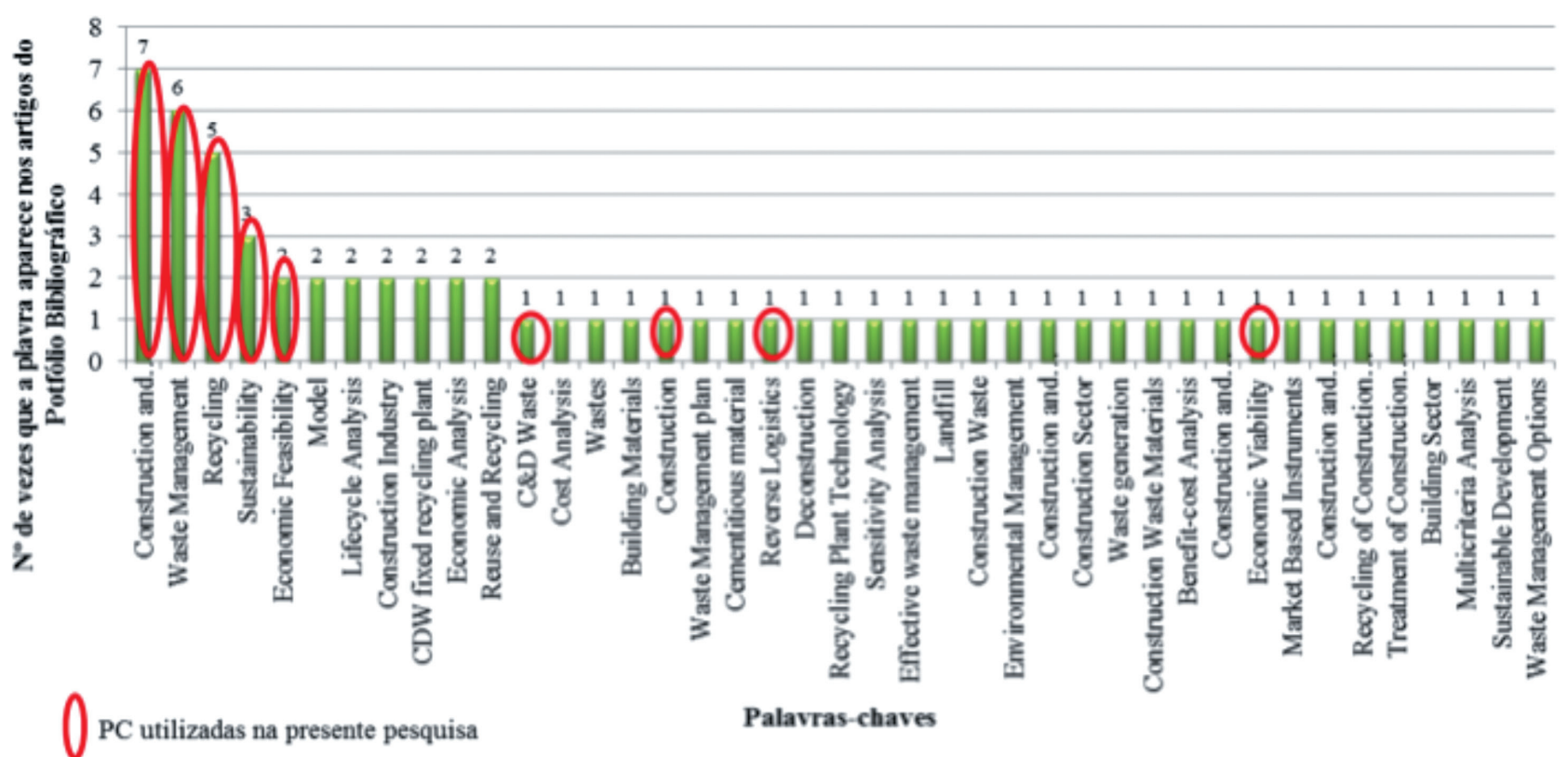

Figura 1: Palavras-chaves mais utilizadas no Portfólio Bibliográfico Fonte: Elaborado pelos autores.

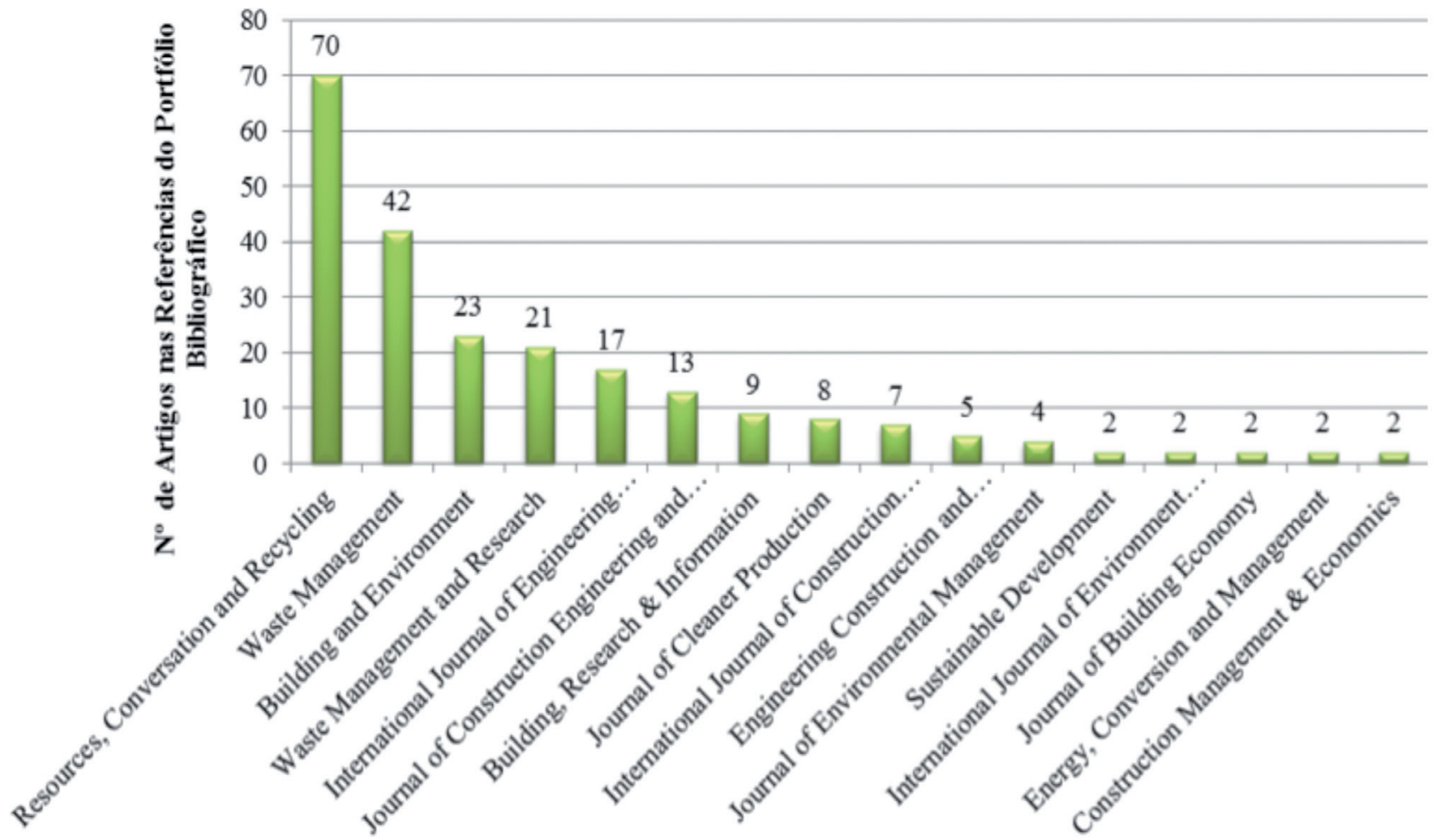

Periódicos

Figura 2: Relevância dos periódicos nas referências dos artigos no Portfólio Bibliográfico Fonte:Elaborado pelos autores. 


\subsubsection{Análise bibliométrica dos artigos do Portfólio Bibliográfico e das referências dos artigos do Portfólio Bibliográfico}

Objetivando-se analisar a relevância dos periódicos presentes nos artigos e referências da pesquisa (Figura 3), considerou-se dois aspectos: o número de artigos do Portfólio Bibliográfico presentes em cada periódico e o número de artigos das referências do Portfólio Bibliográfico presentes em cada periódico.

A partir desse gráfico definiu-se duas linhas de corte para delimitação dos quadrantes de relevância. Esse corte delimita aproximadamente $25 \%$ dos artigos de destaque segundo os aspectos de análise de cada um dos eixos do gráfico.

Novamente, o periódico de maior destaque é o Resources, Conservation and Recycling, visto que este possui um número considerável de artigos no Portfólio Bibliográfico (eixo horizontal) e também muitos artigos citados nas referências (eixo vertical), seguido pelo periódico Waste Management.

Por fim, a Figura 4 apresenta os autores de destaque do Portfólio Bibliográfico, considerando tanto o número de artigo desse autor no Portfólio Bibliográfico quanto o número de artigos do autor do Portfólio Bibliográfico nas referências do Portfólio Bibliográfico. Novamente, foi considerado o corte de $25 \%$ em cada eixo. Aqui percebe-se que não houve nenhum autor que tenha se destacado tanto no Portfólio Bibliográfico quanto nas suas referências. Dois autores se destacaram no Portfólio Bibliográfico: Brito, J. e Coelho, A. com três artigos cada, como pode ser visualizado no $2^{\circ}$ quadrante. No $3^{\circ}$ quadrante encontram-se os autores relevantes para o tema da pesquisa: todos têm até dois artigos presentes no Portfólio Bibliográfico e até sete artigos nas referências do Portfólio Bibliográfico. Por fim, no quarto quadrante tem-se o autor de destaque nas referências do Portfólio Bibliográfico: Tam, V. W. T., com 21 citações nos artigos do Portfólio Bibliográfico.

\subsection{Análise Sistêmica}

Uma vez definidas as lentes de pesquisa na seção dos procedimentos metodológicos, as subseções seguintes apresentarão os resultados da revisão sistêmica da literatura para cada uma das 4 lentes.

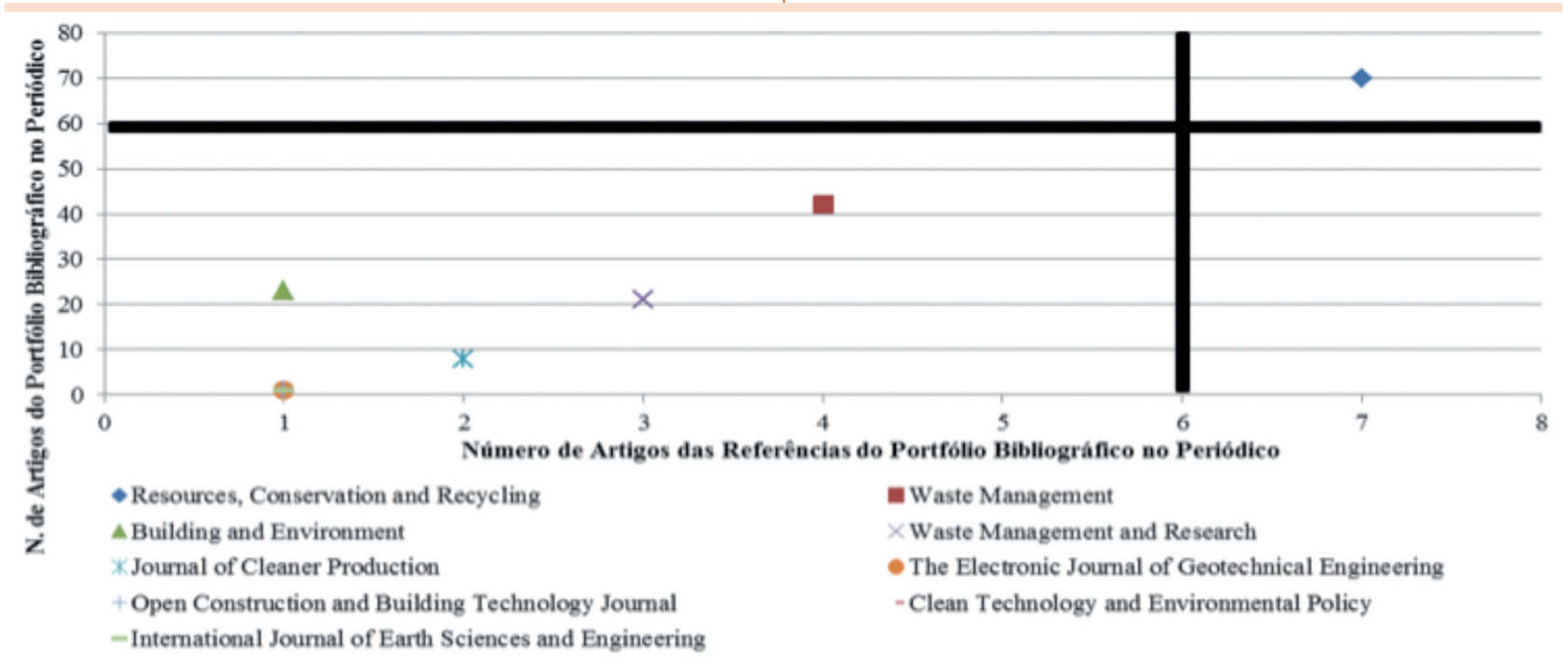

Figura 3: Relevância dos Periódicos presentes nos Artigos e Referências da Pesquisa Fonte: Elaborado pelos autores. 


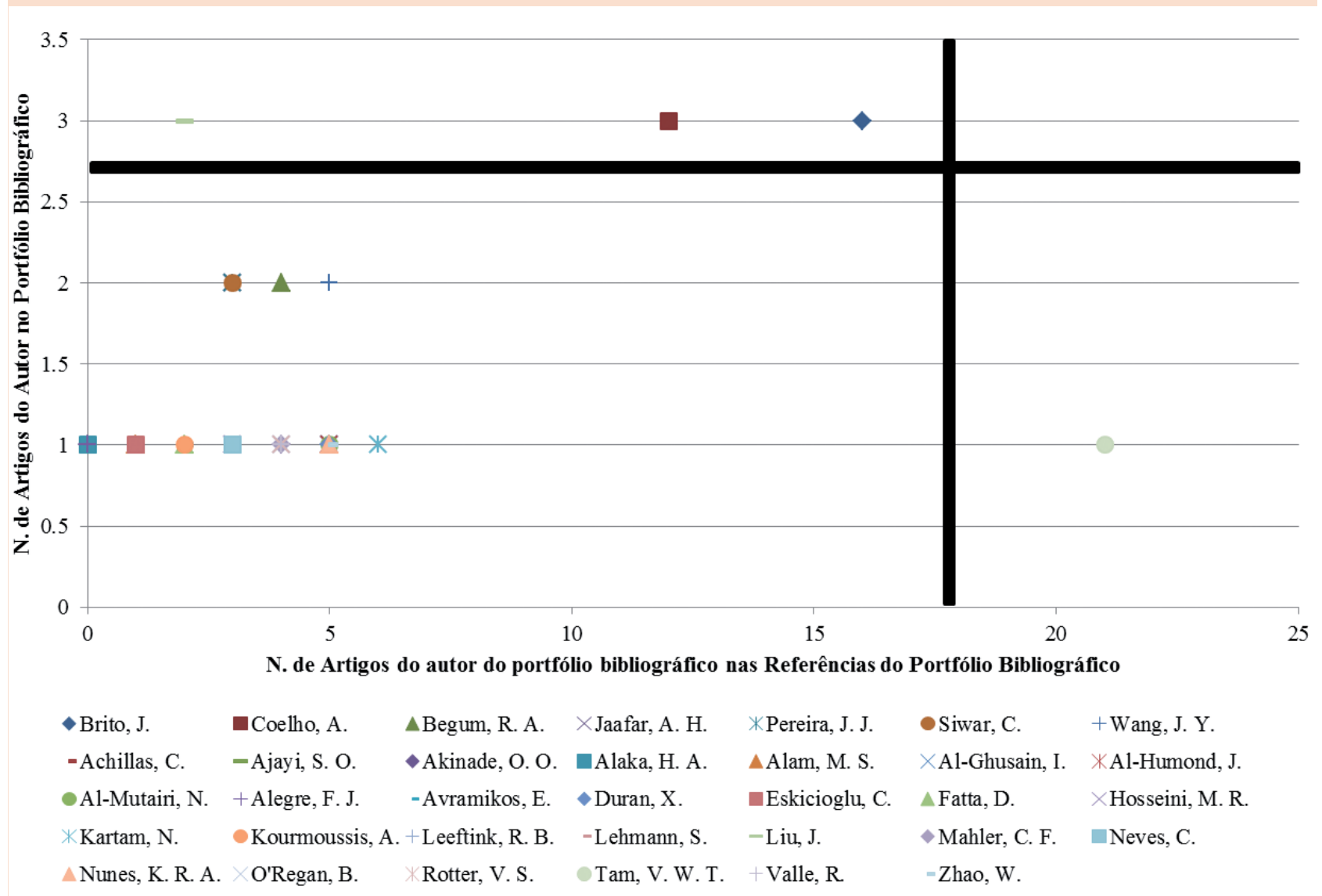

Figura 4: Autores de Destaque do Portfólio Bibliográfico

Fonte: Elaborado pelos autores.

\subsubsection{Lente 01: Natureza e Abordagem da pesquisa}

Essa primeira lente tem como objetivo analisar quais os tipos de pesquisa mais comumente utilizados na área e quais são as principais abordagens feitas pelos pesquisadores. Quanto aos tipos de pesquisa utilizados pelos autores dos artigos do Portfólio Bibliográfico, buscou-se identificar se os artigos eram do tipo exploratório, descritivo, exploratório/descritivo ou se o mesmo não foi explicitado. Dos 21 artigos que compõe o Portfólio Bibliográfico, verificou-se que em nove deles a pesquisa foi do tipo exploratória, em quatro foi descritiva e em sete artigos a pesquisa ocorreu de forma exploratória/descritiva, apenas em um artigo não ficou explicitado o tipo de pesquisa utilizado.
Já no que diz respeito à abordagem da pesquisa, verificou-se que apenas um artigo fez uso de abordagem qualitativa para obter seus resultados, enquanto doze artigos se utilizaram da abordagem quantitativa e quatro abordaram tanto quali quanto quantitativamente o problema da pesquisa. Os outros quatro artigos não se utilizaram de nenhum tipo de abordagem por se tratarem de artigos de revisão de literatura que não incluía coleta de dados.

A análise sistêmica dessa primeira lente permite observar que a pesquisa exploratória quantitativa é a preferida dentre os pesquisadores, isso se deve em muito ao tema da pesquisa, mas também é possível verificar a oportunidade de contribuir, em trabalhos vindouros, com pesquisas que tra- 
balhem o tema de forma exploratória/descritiva e o abordem tanto quali quanto quantitativamente.

\subsubsection{Lente 02: Método de pesquisa}

A segunda lente trata dos métodos de pesquisa utilizados nos artigos do Portfólio Bibliográfico. Os principais métodos encontrados foram o estudo de caso (11 artigos) e a pesquisa survey ( 3 artigos). Os demais trabalhos ou utilizaram outro método de pesquisa ou não utilizaram método nenhum.

O método do estudo de caso se mostrou o preferido dentre os pesquisadores e isso se justifica em virtude do tema da pesquisa. Conforme destacam Coelho \& Brito (2013a), a pesquisa em torno do tema viabilidade econômica de investimento e gestão de uma usina de reciclagem tem características regionais, dependente de muitos fatores físicos, econômicos e sociais do local em que esta será inserida, de forma que as pesquisas devem, preferencialmente, considerar esse aspecto como uma parte relevante da mesma.

\subsubsection{Lente 03: Metodologia}

A terceira lente visa analisar os métodos de viabilidade econômico-financeira determinísticos e/ou estocásticos utilizados nos artigos do Portfólio Bibliográfico. Aqui, busca-se verificar quantos trabalhos utilizaram os indicadores da Metodologia Multi-índice (MMI) proposta por Souza e Clemente (2008), tais como Valor Presente Líquido (VPL), Taxa Interna de Retorno (TIR) e/ou Payback para examinar a viabilidade econômica, se houve utilização da técnica da Análise de Sensibilidade, incorporada à MMI por Lima, Trentin, Oliveira, Batistus \& Setti (2015) e da Análise de Cenários como complementos. Também se buscou verificar se foi considerada a incerteza no processo de decisão por meio da Simulação de Monte Carlo.
Dos 21 artigos, dois trabalhos utilizaram os indicadores VPL, TIR e/ou Payback como metodologia (Zhao et al., 2010; Srour et al., 2013), encontrou-se um único caso de emprego da Análise de Sensibilidade (Coelho \& Brito, 2013b) e quatro trabalhos empregaram a análise de cenários em seus estudos (Coelho \& Brito, 2012; Nunes et al., 2007; Wang et al.,2004 e Duran et al., 2006).

A partir da análise sistêmica gerada por essa lente, verifica-se uma lacuna na literatura no que tange à metodologia empregada nos trabalhos. Dentro do Portfólio Bibliográfico há vários artigos que buscam analisar a viabilidade econômico-financeira de se investir em uma URRCD, porém, em sua maioria, essa análise da viabilidade ocorre apenas considerando custos e receitas do investimento (Gegum et al., 2006; Liu \& Wang, 2013; Yeheyis et al., 2013). Não há uma análise aprofundada de quase nenhum indicador de viabilidade econômico-financeira, bem como quase é desconsiderada a questão dos riscos e do retorno do investimento. Nenhum trabalho considerou em sua análise a Teoria das Opções Reais ou a Flexibilidade Gerencial, consideradas como indicadores importantes para avaliar a viabilidade do investimento na URRCD. Houve ocorrência de quatro estudos que fizeram estudo de caso (Kasthurba et al., 2014; Tam et al., 2009; Nagalli, 2013 e Rodriguez et al., 2007), porém nenhum deles fez um diagnóstico mais completo da viabilidade econômico-financeira das plantas em estudo.

Dessa forma, essa lente se mostrou como a mais importante para a presente análise sistêmica, uma vez que permitiu verificar que há uma lacuna na literatura quanto ao uso de metodologias de viabilidade econômica, em virtude de os trabalhos não terem se utilizado da combinação de vários indicadores da MMI combinados com as análises de cenário e de sensibilidade e a flexibilidade gerencial. Assim, um estudo que combine a maior gama possível de indicadores se mostra necessário, prin- 
cipalmente objetivando encontrar resultados os mais próximos possíveis da realidade.

\subsubsection{Lente 04: Gestão e Sustentabilidade}

Enquanto a lente 03 buscou entender os métodos de viabilidade econômico-financeira dos artigos que abordaram a questão da viabilidade econômico-financeira, a presente lente está mais focada em verificar, nos artigos que trataram da gestão e sustentabilidade dos resíduos da construção e demolição, se houveram propostas de novas formas de analisar o processo de gestão e sustentabilidade. Seis artigos utilizaram de outras abordagens para analisar a gestão das usinas, as quatro principais foram: análise multicritério (Banias et al., 2010), regressão logística (Begum et al., 2009), análise do ciclo de vida (Yeheyis et al., 2013) e análise do custo-benefício (Gegum et al., 2006).

Porém, esses artigos se restringem, em sua maioria, a analisar unicamente casos isolados de gestão e não integraram a análise de sustentabilidade nem analisaram a viabilidade econômica. Dessa forma, essa lente permite verificar que há, de fato, uma lacuna nos estudos que compõe o Portfólio Bibliográfico. Percebe-se que há espaço para que se estude tanto a viabilidade econômicofinanceira da implantação de uma URRCD, quanto sua gestão e sustentabilidade.

\section{Considerações finais}

O presente trabalho tinha como objetivo geral identificar lacunas passíveis de se tornarem futuras pesquisas a respeito da gestão, sustentabilidade e viabilidade econômico-financeira da implantação de uma URRCD privada para a destinação correta dos RCD. Para atingir esse objetivo, foi utilizada a metodologia ProKnow-C, que busca selecionar os principais artigos a respei- to do tema de pesquisa de forma a construir um Portfólio Bibliográfico.

De posse do Portfólio Bibliográfico, foram feitas as análises bibliométrica e sistêmica do mesmo. A análise bibliométrica mostrou que os periódicos que mais publicam a respeito do tema são Resources, Conservation and Recycling e Waste Management. Verificou-se que apenas sete autores tiveram dois ou três artigos presentes no Portfólio Bibliográfico, mostrando que a pesquisa é diversificada. Contudo, a análise cruzada entre os artigos do Portfólio Bibliográfico e os artigos referenciados nesses trabalhos mostrou que o autor V. W. T. Tam, é o de maior destaque, por ter 21 artigos citados no Portfólio Bibliográfico.

Por fim, a análise sistêmica, por intermédio de suas lentes, buscou identificar os destaques e as lacunas dos artigos do Portfólio Bibliográfico. Por meio das quatro lentes propostas nesse trabalho, verificou-se que a abordagem quantitativa foi a mais utilizada nos artigos do Portfólio Bibliográfico, sendo, portanto, um dos destaques. Entendeu-se que o Estudo de Caso se mostrou como o método mais adotado nos trabalhos, em virtude, principalmente, do tema da pesquisa apresentar características regionais.

A principal lacuna encontrada na análise sistêmica foi a respeito da metodologia. Verificouse que, nos artigos que compõe o Portfólio Bibliográfico, não houve aprofundamento da análise da viabilidade econômico-financeira, não sendo consideradas as dimensões de retorno e riscos, as quais podem afetar diretamente neste tipo de investimento. Também notou-se a falta de outros procedimentos para análise da viabilidade econômico-financeira, tais como: Análise de Sensibilidade, Análise de Cenários, Teoria das Opções Reais e Fluxo Gerencial. Dessa forma, essa lente se mostrou como a mais importante para a presente análise sistêmica. Logo, um estudo que empregue vários indicadores se mostra necessário, 
principalmente objetivando verificar quais métodos e indicadores são mais importantes e melhor condizem com a realidade.

Por fim, conclui-se que o presente trabalho atingiu seu objetivo ao apresentar um Portfólio Bibliográfico com as mais importantes publicações a respeito do tema de estudo, as quais possivelmente servirão de base para referenciais teóricos de futuros trabalhos acadêmicos e científicos, tendo assim cumprido seu papel como contribuição prática. No que se refere à contribuição acadêmica, este artigo utilizou o método ProKnow-C, apresentado todos os passos do mesmo, portanto, ele também serve como base para futuros pesquisadores que tenham como objetivo aplicar esse mesmo método em suas pesquisas, mesmo que em outras áreas. Quanto à limitação da pesquisa, o presente artigo não apresentou uma análise aprofundada específica para cada um dos artigos que compõe o Portfólio Bibliográfico, o que impede uma identificação mais densa das lacunas de pesquisa nessa área, fica, portanto, a sugestão de que, em um trabalho futuro, seja feita essa análise, como forma de qualificar a pesquisa.

\section{Referências}

Ajayi, S. O., Oyedele, L. O., Bilal, M., Akinade, O. O., Alaka, H. A., Owolabi, H. A., \& Kadiri, K. O. (2015). Waste effectiveness of the construction industry: Understanding the impediments and requisites for improvements. Resources, Conservation and Recycling, 102, 101-112.

Alves, A. L., \& Ensslin, L. (2012). Avaliação de curso técnico: uma metodologia estruturada para construir um portfólio bibliográfico atualizado. Revista Espacios, 37(7), 1-24.

Associação Brasileira de Empresas de Limpeza Pública e Resíduos Especiais - ABRELPE. (2014). Panorama nos resíduos sólidos no Brasil: 2014, São Paulo, SP, Brasil.

Azevedo, G. O. D. D., Kiperstok, A., \& Moraes, L. R. S. (2006). Resíduos da construção civil em Salvador: os caminhos para uma gestão sustentável. Engenharia Sanitária e Ambiental, 11(1), 65-72.
Banias, G., Achillas, C., Vlachokostas, C., Moussiopoulos, N., \& Tarsenis, S. (2010). Assessing multiple criteria for the optimal location of a construction and demolition waste management facility. Building and Environment, 45(10), 2317-26.

Begum, R. A., Siwar, C., Pereira, J. J., \& Jaafar, A. H. (2006). A benefit-cost analysis on the economic feasibility of construction waste minimization: the case of Malaysia. Resources, Conservation and Recycling, 48(1), 86-98.

Begum, R. A., Siwar, C., Pereira, J. J., \& Jaafar, A. H. (2009). Attitude and behavioral factors in waste management in the construction industry of Malaysia. Resources, Conservation and Recycling, 53(6), 321-328.

Bortoluzzi, S. C., Ensslin, S. R., \& Ensslin, L. (2011). Avaliação de desempenho em redes de pequenas e médias empresas: Estado da arte para as delimitações postas pelo pesquisador. Estratégia \& Negócios, 4(2), 202-222.

Bortoluzzi, S. C., Ensslin, S. R., \& Ensslin, L., Chaves, L. C. (2014). Indicadores de desempenho propostos em pesquisas nacionais e internacionais para avaliar redes de pequenas e médias empresas (PMEs). Revista Espacios, 35(2), 1-18.

Coelho, A., \& Brito, J. (2012). Influence of construction and demolition waste management on the environmental impact of buildings. Waste Management, 32(3), 532-541.

Coelho, A., \& Brito, J. (2013a). Economic viability analysis of a construction and demolition waste recycling plant in Portugal - part I: location, materials, technology and economic analysis. Journal of Cleaner Production, $39,338-352$.

Coelho, A., \& Brito, J. (2013b). Economic viability analysis of a construction and demolition waste recycling plant in Portugal - part II: economic sensitivity analysis. Journal of Cleaner Production, 39, 329-337.

Duran, X., Lenihan, H., \& O'regan, B. (2006). A model for assessing the economic viability of construction and demolition waste recycling - the case of Ireland. Resources, Conservation and Recycling, 46(3), 302-320.

ENDNOTE. Disponível em: <https://www. myendnoteweb.com/>. Acesso em: Set. 2015.

Ensslin, L., Ensslin, S. R., Lacerda, R. T. O., \& Tasca, J. E. (2010). Proknow-C, Knowledge Development Process - Constructivist. Processo técnico com patente de registro pendente junto ao INPI. Brasil, 2010.

Ensslin, L., Ensslin, S. R., \& Souza, M. V. (2014). Gerenciamento de portfólio de produtos na indústria: Estado da arte. Revista Produção Online, 14(3), 790821.

Fatta, D., Papadopoulos, A., Avramikos, E., Sgourou, E., Moustakas, K., Kourmoussis, F., \& Loizidou, M. (2003). Generation and management of construction and demolition waste in Greece - an existing challenge. Resources, Conservation and Recycling, 40(1), 81-91. 
Google Scholar. Disponível em: <http://scholar.google. com.br/>. Acesso em: Set. 2015.

Harzer, J. H. (2015). Indicadores de riscos em projetos de investimentos: uma contribuição à Metodologia Multi-índice. Tese de Doutorado, Pontifícia Universidade Católica do Paraná, Curitiba, PR, Brasil.

Hosseini, M. R., Rameezdeen, R., Chileshe, N., \& Lehmann, S. (2015). Reverse logistics in the construction industry. Waste Management \& Research, 33(6), 499-514.

Kartam, N., Al-Mutairi, N., Al-Ghusain, I., \& AlHumoud, J. (2004). Environmental management of construction and demolition waste in Kuwait. Waste Management, 24(10), 1049-59.

Kasthurba, A., Reddy, K., \& Reddy, D. V. (2014). Sustainable Approaches for Utilizing Waste in Building Construction: Two Case Studies in India. International Journal of Earth Sciences and Engineering, 7(3), 838-844.

Knoff, L. C., Lacerda, R. T. O., Ensslin, L., \& Ensslin, S. R. (2014). Mapeamento de publicações científicas sobre estratégia de manufatura: uma abordagem em processos. Revista Produção Online, 14(1), 403-429.

Lacerda, R. T. O., Ensslin, L., \& Ensslin, S. R. (2012). Uma análise bibliométrica da literatura sobre estratégia e avaliação de desempenho. Gestão \& Produção, 19(1), 59-78.

Lima, J. D. de, Trentin, M. G., Oliveira, G. A., Batistus, D. R., \& Setti, D. (2015). A systematic approach for the analysis of the economic viability of investment projects. International Journal of Engineering Management and Economics, 5(1/2), 19-34.

Liu, J., \& Wang, Y. (2013). Cost Analysis of Construction and Demolition Waste Management: Case Study of the Pearl River Delta of China. Open Construction and Building Technology Journal, 7, 251-263.

Marques Neto, J.C. (2005). Gestão de resíduos de construção e demolição no Brasil. São Carlos: RiMa, $162 \mathrm{p}$.

Nagalli, A. (2013). The Sustainability of Brazilian Construction and Demolition Waste Management System. The Electronic Journal of Geotechnical Engineering, 18, 1755-59.

Nagaoka, M. P. T., Ensslin, L., Ensslin, S. R., \& Nagaoka, A. K. (2011). Gestão de propriedades rurais: Processo estruturado de revisão de literatura e análise sistêmica. Revista Brasileira de Agrociência, 17(4), 410 - 419.

Nunes, K. R. A., Mahler, C. F., Valle, R., \& Neves, C. (2007). Evaluation of investments in recycling centers for construction and demolition wastes in Brazilian municipalities. Waste Management, 27(11), 1531-1540.

Pinto, T. P. (1999). Metodologia para a gestão diferenciada de Resíduos sólidos da construção urbana. Tese de Doutorado, Escola Politécnica da Universidade de São Paulo, São Paulo, SP, Brasil.
Rodríguez, G., Alegre, F. J., \& Martínez, G. (2007). The contribution of environmental management systems to the management of construction and demolition waste: The case of the Autonomous Community of Madrid (Spain). Resources, Conservation and Recycling, 50(3), 334-349.

Santos, F. R., \& Pompeu, R. B. (2014). Logística reversa de resíduos da construção civil: uma análise de viabilidade econômica. Revista Tecnológica da Fatec Americana, 2(1), 105-20.

Semler, R. F., Bortoluzzi, S. C., \& Schenatto, F. J. A. (2015). Avaliação de desempenho da inovação em redes de empresas: análise bibliométrica e sistêmica da literatura científica internacional. Revista Espacios, 36(24), 1-14.

Souza, A., \& Clemente, A. (2008). Decisões Financeiras e Análise de Investimentos: Fundamentos, técnicas e aplicações. 6 ed. São Paulo: Atlas.

Srour, I. M., Chehab, G. R., El-Fadel, M., \& Tamraz, S. (2013). Pilot-based assessment of the economics of recycling construction demolition waste. Waste Management \& Research, 31(11), 1170-1179.

Tam, V. W., Kotrayothar, D., \& Loo, Y. C. (2009). On the prevailing construction waste recycling practices: a South East Queensland study. Waste Management \& Research, 27(2), 167-174.

Wang, J. Y., Touran, A., Christoforou, C., \& Fadlalla, H. (2004). A systems analysis tool for construction and demolition wastes management. Waste management, 24(10), 989-997.

Yeheyis, M., Hewage, K., Alam, M. S., Eskicioglu, C., \& Sadiq, R. (2013). An overview of construction and demolition waste management in Canada: a lifecycle analysis approach to sustainability. Clean Technologies and Environmental Policy, 15(1), 81-91.

Zhao, W., Leeftink, R. B., \& Rotter, V. S. (2010). Evaluation of the economic feasibility for the recycling of construction and demolition waste in China - The case of Chongqing. Resources, Conservation and Recycling, 54(6), 377-389.

Recebido em 22 mai. 2017 / aprovado em 30 nov. 2017

Para referenciar este texto

Gularte, L. C. P., Bortoluzzi, S. C., Lima, J. D., Pinto, M. A. N., \& Goffi, A. S. Análise bibliométrica e sistêmica da literatura sobre viabilidade econômicafinanceira, gestão e sustentabilidade da reciclagem de resíduos da construção civil. Exacta, São Paulo, v. 16, n. 4, p. 45-58. out./dez. 2018. Disponível em: <https://doi.org/10.5585/ExactaEP.v16n4.7436> 